\title{
New Broadband LIDAR for Greenhouse Carbon Dioxide Gas Sensing in the Earth's Atmosphere
}

\author{
Elena Georgieva ${ }^{1,2^{*}}$, William S. Heaps ${ }^{2}$, Wen Huang ${ }^{2,3}$ \\ 'Joint Center for Earth Systems Technology/UMBC \\ ${ }^{2}$ NASA Goddard Space Flight Center \\ ${ }^{3}$ Science Systems and Applications
}

\begin{abstract}
We present airborne measurements of a novel eye-safe spectrally broadband LIDAR capable of dealing with the atmospherically-induced variations in $\mathrm{CO}_{2}$ absorption using a Fabry-Perot based detector. The Fabry-Perot solid etalon in the receiver part is tuned to match the wavelength of several $\mathrm{CO}_{2}$ absorption lines simultaneously. The broadband technique tremendously reduces the requirement for source wavelength stability, instead putting this responsibility on the Fabry-Perot based receiver. The instrument technology we are developing has a clear pathway to space and realistic potential to become a robust, low risk space measurement system.

Keywords: Instrumentation, measurement and metrology (120.0120), remote sensing (280.0280), atmospheric composition $(010.1280)$, optical instruments $(120.4640)$, absorption $(300.1030)$, interferometry (120.3180), Fabry-Perot (120.2230)
\end{abstract}

\section{INTRODUCTION}

There is a pressing need for measurements of atmospheric carbon dioxide concentration with high spatial and temporal resolution for global and regional studies of the carbon cycle. Such measurements will better resolve the linkage between global warming and anthropogenic $\mathrm{CO}_{2}$ emissions. In the Decadal Survey 
of Earth Science the National Research Council recommended that NASA develop, build, and fly a laserbased system for precision measurement of total carbon dioxide (the ASCENDS mission). The mission demands measurements of $\mathrm{CO}_{2}$ to a precision of $1 \mathrm{ppm}$ out of the total $\sim 400 \mathrm{ppm}$ column in order to locate sources and sinks. Achieving that is difficult due to a strong dependence on changes in atmospheric pressure and temperature of atmospheric carbon dioxide absorption line position, shape, and strength. Our team at Goddard Space Flight Center (GSFC) is developing a LIDAR system for column $\mathrm{CO}_{2}$ measurement based on an innovative new technique employing a spectrally broad laser source centered at $1573.3 \mathrm{~nm}$. The backscattered light of the broadband illumination beam is detected and spectrally resolved using Fabry-Perot based receiver.

\section{MEASUREMENT APPROACH}

Various approaches have been used to detect trace gases in the atmosphere. Most of them are currently under development as candidates for the ASCENDS mission and require multiple lasers operating at different, very narrow bandwidth wavelengths. Our broadband lidar (BBL) is capable of mitigating inaccuracy associated with atmospherically-induced variations in $\mathrm{CO}_{2}$ absorption line shape and strength using only one laser. We demonstrated our new approach using the $\mathrm{CO}_{2}$ measurements from the NASA DC-8 aircraft in July and August, 2011 over different locations.

The BBL transmitter consists of Nd:YAG laser (Surelite: IPG Photonics model number EAR-10K-1571-LPSF) and an OPO system with a maximum spectral bandwidth covering the $1572.2 \mathrm{~nm}$ to $1574.2 \mathrm{~nm}$ range. The laser operates in pulsed mode, emitting $6 \mathrm{~ns}$ pulses over a $2 \mathrm{~nm}$ spectral range. The reason to choose the OPO technique is because it is a mature technology, highly efficient, gives a possibility to control the signal wavelength, the KTP crystals are commercially available and the transmitter part is compact and easy for alignment. The KTP crystals (United Crystals Company) were X-cut, 4 X 4 X $20 \mathrm{~mm}$, for non-critical phase matching at room temperature for pump $1064 \mathrm{~nm}$, signal $1573 \mathrm{~nm}$ and idler 3288.16-nm wavelengths.

The $1064 \mathrm{~nm}$ beam is expanded and combined with a $1573 \mathrm{~nm}$ beam; the two then exit rogether through a nadir-viewing window of the aircraft. The reflection from the ground is collected by a telescope Vixen model VC200L. The beam diameter out of the transmit telescope is $\sim 75 \mathrm{~mm}$, with a divergence of $\sim 3 \mathrm{mrad}$ 
as determined from actual laboratory measurements. Because the $\mathrm{CO}_{2}$ absorption strength is not constant but varies with temperature and pressure, all the ASCENDS candidate lidars employ more than two wavelengths in an effort to untangle those effects. Our bandpass filter is $4 \mathrm{~nm}$ wide. Fig. 1 illustrates the operation of the lidar receiver. The thin blue line shows the transmission of the FP. The thin red lines are the $\mathrm{CO}_{2}$ absorption lines. We are aligned with three $\mathrm{CO}_{2}$ absorption lines $(1573.332 \mathrm{~nm}, 1573.992 \mathrm{~nm}$ and $1573.679 \mathrm{~nm})$. Because our source emits wavelengths that are strongly absorbed by $\mathrm{CO}_{2}$ as well as wavelengths that are only weakly absorbed all in a single laser pulse, we use the receiver portion of our lidar to separate the wavelengths and provide our "differential" absorption.

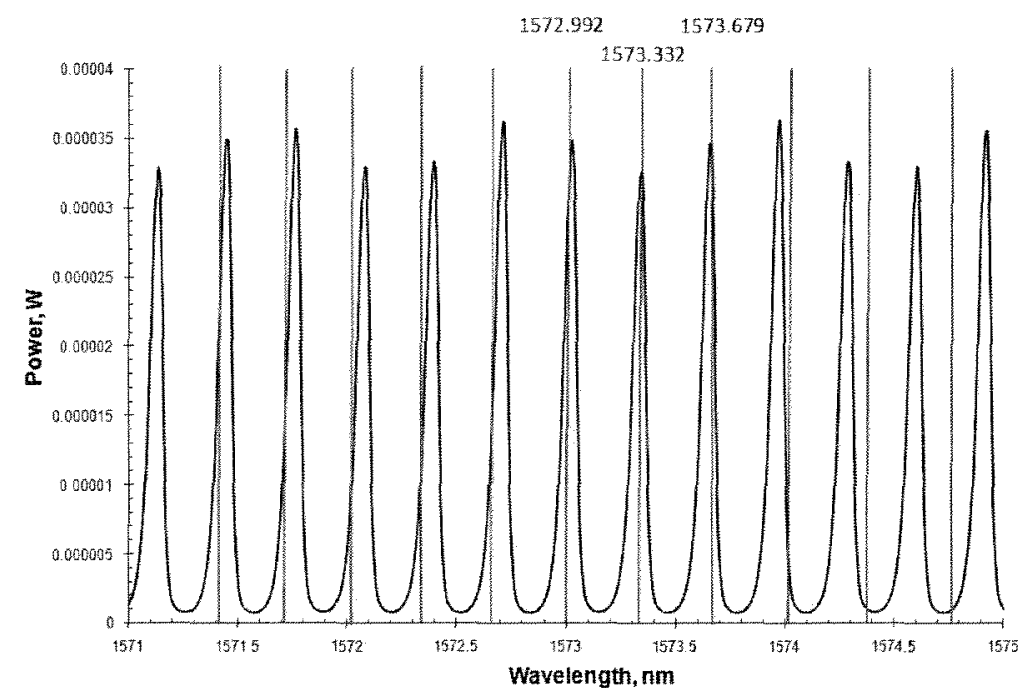

Fig. 1 This depicts the operation of our lidar receiver.
Light passing through the FP bounces back and forth between the two surfaces crating interference with the result that it only transmits light for which integral number of half wavelengths exactly fits between the two surfaces. By changing the thickness of the glass plate, different colors can be

allowed to pass. By changing the reflectivity, the width of the passband can be controlled. We select a reflectivity such that the width of a passband is nearly the same as the width of $\mathrm{CO}_{2}$ absorption and we place the FP inside an oven (Thorlabs Inc.) so that the temperature can be controlled. Changing the temperature changes the optical thickness of our FP and thereby alters the wavelengths that are transmitted. Our lidar receiver has two channels. The FP channel has both the bandpass filter and the FP in front of the detector. Thus it responds mostly to light that ean be absorbed by the $\mathrm{CO}_{2}$. The reference (REF) channel only has the 
bandpass filter in front of the detector. Most of the light that reaches this detector is unaffected by $\mathrm{CO}_{2}$. Fig. 2 is the lidar system with one side of its box removed to show the telescope and receiver section.

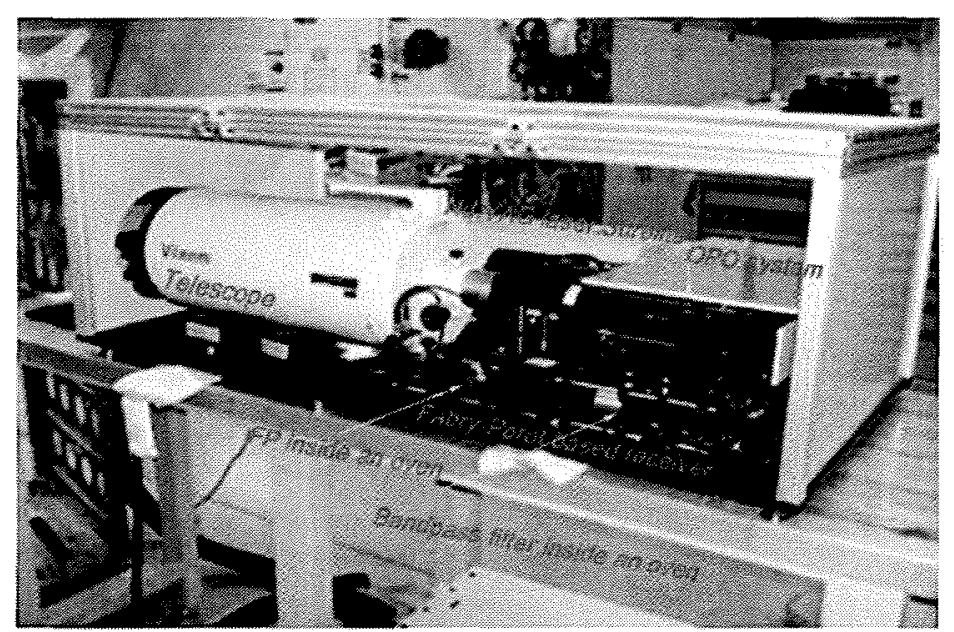

Fig. 2 This picture show the Broad Band lidar just prior to installation in the DC-8. The side covers are removed.
Fig. 3 are the two channels as seen on the screen of Tektronics oscilloscope. The first peak is the initial fire of the laser. This signal serves to monitor the performance of the system and enables corrections for drifts in the detector or the FP temperature etc. The second large peak is the reflected light signal from the ground. Comparing the changes in the

ratios of the FP and REF channel for this signal enables calculation of the $\mathrm{CO}_{2}$ column between the aircraft and the ground. Fig. 4 shows the lidar installed in the cargo bay of the DC-8. In operation the laser fires at a rate of $20 \mathrm{~Hz}$. We record the signals from the FP and REF channels as a function of time and average shots together.

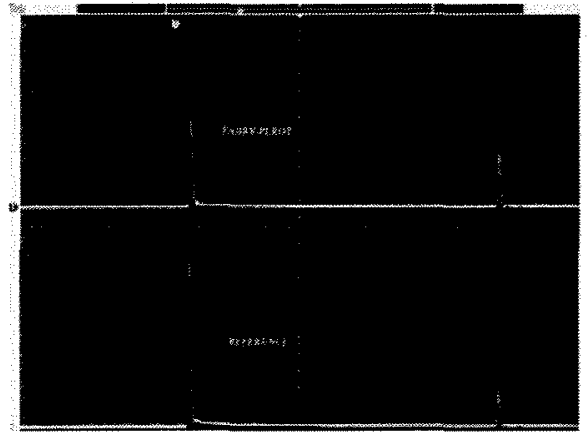

Fig. 3 This shows a typical lidar return signal.

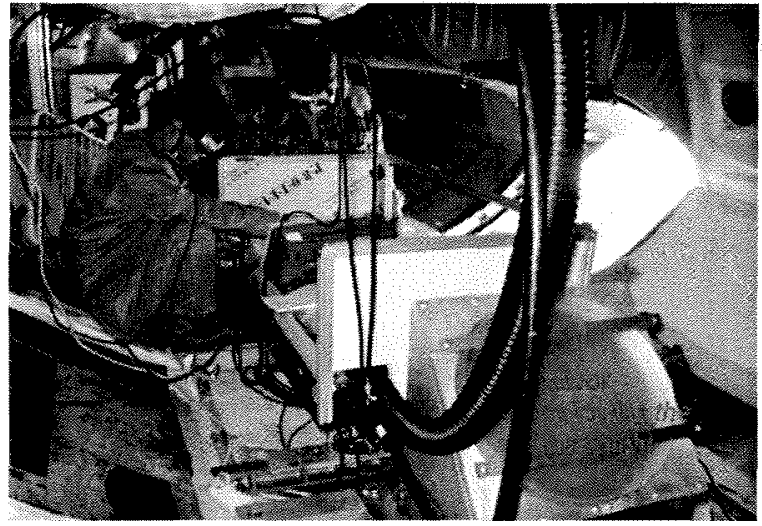

Fig. 4 This is the instrument installed in the cargo bay of the

DC-8. 


\section{DATA ANALYSIS}

The analysis for data obtained on our test flights proceeds from Beer's Law of Absorption which may be stated as follows:

$$
I=I_{0} e^{-n s z}
$$

where $I$ is the intensity of the absorbed light, $I_{0}$ is the intensity before absorption, $n$ is the number density of absorbers, $s$ is the absorption cross section, and $z$ is the range through the absorbing medium. We may also write

$$
\log \frac{I}{I_{0}}=-n s z
$$

which shows that the log of the ratio of the absorbed intensity to the initial intensity is a linear function of the range and the absorber number density. With a DIAL system we essentially measure $I, I_{0}$ and $z$. We presume to have measured the absorption cross section in the laboratory so we can solve for $\mathrm{n}$ which is the number of $\mathrm{CO}_{2}$ per square $\mathrm{cm}$ along the absorption path.

The lidar system measurement of $I_{0}$ involves a number of instrumental factors (eg. percentage of transmitted light sampled, filter and FP transmission, detector response etc.). The measurement of $I$ also involves same factors plus the transmission of the atmosphere, the reflectivity of the ground etc. In principle the instrumental factors could all be measured or calculated but this is difficult to do. Instead we make a second measurement (let's call them $I_{0}^{\star}$ and $I \star$ ) at a second wavelength using the same equipment. Beers law should hold for this second measurement except that $s \star$ will have a different value. Subtracting the two equations yields

$$
\log \frac{I}{I_{0}}-\log \frac{I^{\star}}{I_{0}^{\star}}=-n\left(s-s^{\star}\right) z
$$

For this measurement all the instrumental factors in the measurement divide out and if we choose the two wavelengths so that ground reflectivity and atmospheric transmission are the same (except for absorption by $\mathrm{CO}_{2}$ ) then our raw measurement of the difference of the two ratios is the same as the actual difference of the two ratios. If we know the difference between the two absorption coefficients we can solve for carbon dioxide abundance. The difference between the absorption coefficients can be measured in the lab, however 
in the real atmosphere they are not constants but depend on pressure and temperature. From our knowledge of the behavior of spectroscopic line shapes with temperature and pressure we can deduce the correct values of these absorption coefficients if we make a few additional measurements at other wavelengths. Because the measurements for the broad band lidar are made over a range of wavelengths the absorption cross sections in our analysis are weighted averages over a span of wavelengths. Essentially our $s$ is an integral of the $\mathrm{CO}_{2}$ absorption over all three lines multiplied by the transmission of the FP and the bandpass filter and $s \star$ is an integral of the $\mathrm{CO}_{2}$ absorption multiplied by the transmission of the BP filter alone. By judicious choice of the BP filter and FP transmission characteristics it is possible to reduce very significantly the effects of pressure and temperature on these average cross sections. One of the strengths of the BBL as we implement it is that the same detector, filter, and FP are used to measure $I_{0}$ as we use to measure $I$. In this way we are immune to 'drifts' in the components of the system.

These average cross sections could in principle be calculated by measurements of the BP filter and FP transmission and using cross sections for $\mathrm{CO}_{2}$ obtained in the lab. They could also be determined by calibrating the whole instrument in the lab introducing known amounts of $\mathrm{CO}_{2}$ into the absorption path of the instrument and noting the ratio response. We will use both methods for precise final calculations. For this paper we have adopted a different approach. The purpose of our recent test flights was to demonstrate the measurement capability of the broadband lidar and to investigate its characteristics. We already know that the approximate column abundance of $\mathrm{CO}_{2}$ is about $390 \mathrm{ppm}$. Using the pressure and aircraft altitude measurements from the DC-8 along with the range information from the lidar we can calculate what fraction of the total airmass the lidar has sounded. Multiplying the fraction by 390 give the approximate amount of $\mathrm{CO}_{2}$ that the instrument has sensed. Comparing this number to the signal response of the lidar permits the calculation of the difference in absorption coefficients for the FP and REF channels. If we plot the log of the FP/REF ratio versus the amount of $\mathrm{CO}_{2}$ sensed (essentially the product of $n$ and $z$ ) then the result should be a line with a slope equal to this difference of average absorption coefficients (s- $\left.S^{*}\right)$. The next section shows the progression of the data analysis to achieve this plot. 


\section{DC-8 MAJOR ASCENDS FLIGHT TEST CAMPAIGN JULY/AUGUST,}

\section{1, PALMDALE, CA; PRELIMINARY DATA}

Prior to the flights we performed tests on tuning the FP in the receiver. On July 22 we did night measurements while shooting at a $3.1 \mathrm{~km}$ range and changing the oven temperature with the FP. The Fig.5

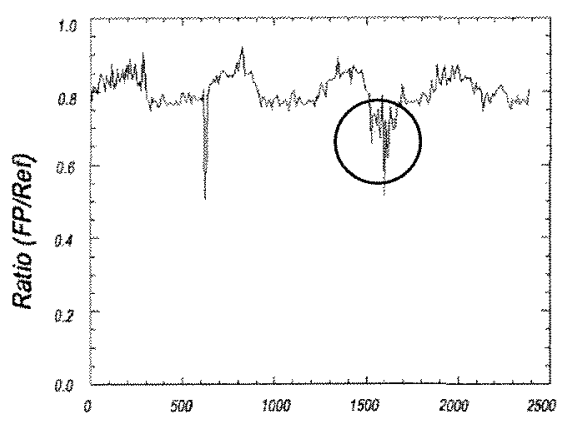

Figure 5. We put the FP etalon through several temperature changes. Lowest ratio shows the best temperature (system sensitive to $\mathrm{CO}_{2}$ ) shows how the ratio changes with temperature of the etalon and the optimal temperature for the system. The circle is the region where the ratio has a minimum (corresponding to maximum sensitivity, FP transmission bands are aligned with the three strong $\mathrm{CO}_{2}$ absorption lines) at the temperature $50.6 \mathrm{deg} \mathrm{C}$. Noise in the data is due to the strong wind moving the mirror reflecting the laser beam.

During an instrument-validation flight over central

California, the DC-8 flew over the Rail Road Valley on August 3. When the plane is landing, ratio is going up which means less and less absorption of carbon dioxide (smaller atmospheric column). The FP signal is increasing and as a result the ratio of $\mathrm{FP} / \mathrm{Ref}$ is increasing as well. (Fig 6). The ratio changed by 0.6 for $4.3 \mathrm{~km}$. At the shortest ranges shown the lidar signal is very large resulting in saturation of the detectors.

Because the location of the broadband lidar

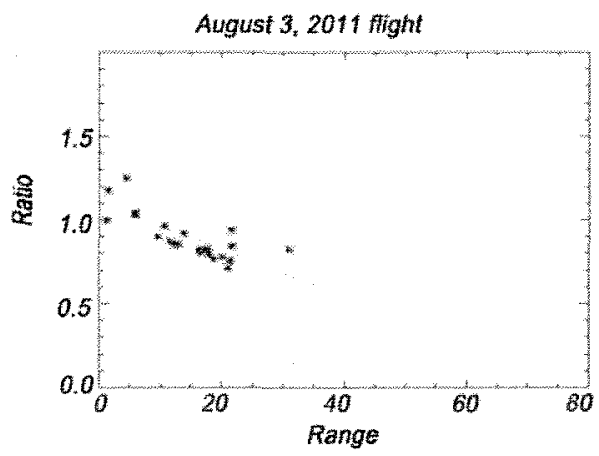

Figure 6 shows the change in ratio with altitude of the plane. The range is in microseconds; 1 microsecond is 150 m. 40 microseconds $=6000 \mathrm{~m}=6 \mathrm{~km} .20$ microseconds $=$ $3000 \mathrm{~m}=3 \mathrm{~km}$. At $6 \mathrm{~km}$ the ratio is $\sim 0.5$ and at $1.7 \mathrm{~km}$ it change up to 1.1 .

was in the cargo pit of the DC- 8 airplane

during flights we experienced a huge temperature changes which influenced the precision of the measurements. 


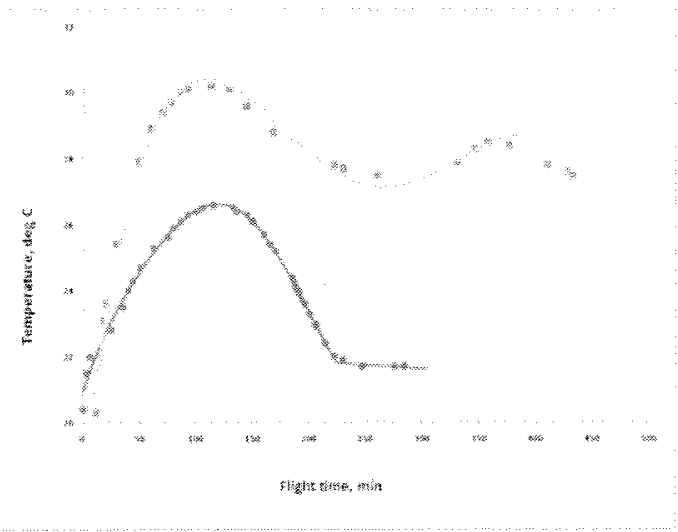

Figure7. The plot shows temperature changes in the pit on two different flights - on August 3 (pink) and August 7 (yellow).
We observed temperature changes of over $10 \mathrm{deg}$ C during the flights (Fig. 7). Various parts of the telescope cool at different rates as some are better conductors and have different rates of expansion. The back focus of the telescope can move approximately $0.25 \mathrm{~mm}$ for every $1^{\circ} \mathrm{C}$ change in temperature. This change in focus due to temperature is a serious problem. On one of the flights we monitored a lot of moisture in the cargo pit causing condensation on the optics. Signals dropped down because of the water on the nadir

viewing window and the near field ratio changed. We excluded these data points from the analysis. Huge temperature changes may also affect the KTP crystals performance because the laser peak moves with temperature (Fig 8). For that experiment we used an OSA (Optical spectrum analyzer) to monitor the lidar peak while changing KTP temperature.

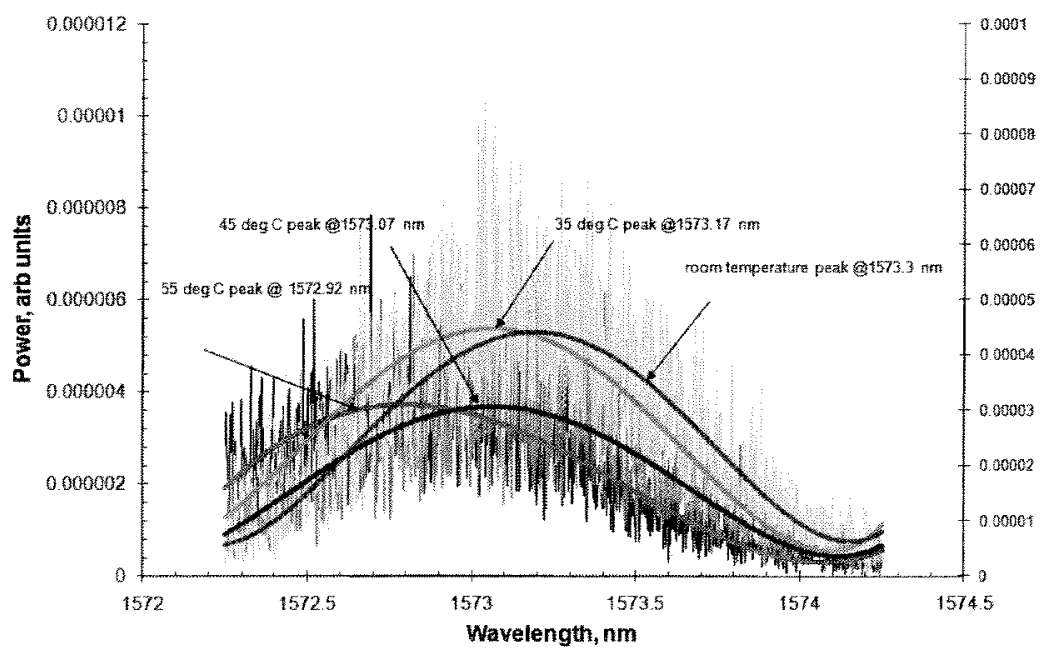

Figure 8. Plot shows how the lidar peak changes position with changing the temperature of KTP crystals. The peak moves to smaller wavelengths with increasing the crystals temperature. 
The DC-8's flight track is shown in red for its flight on Tuesday August 9, 2011 to Tucson and back to Palmdale, CA. During the flight, the broadband lidar instrument was tested at variety of altitudes and over different surface terrains. Figure shows the change in ratio during descent.
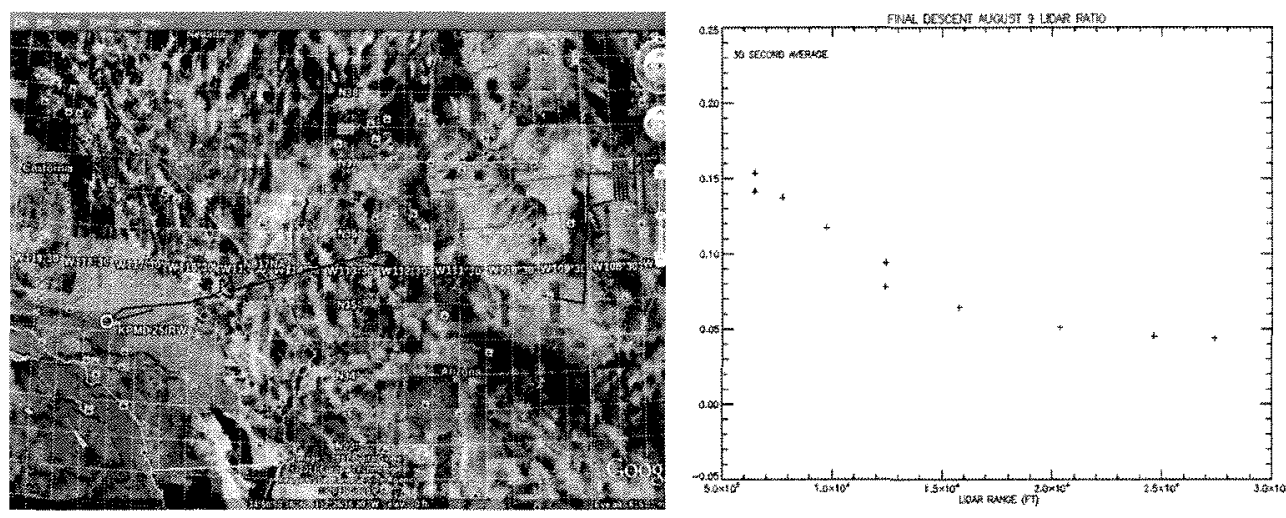

Figure 9 As the altitude of the plane is changing, the ratio changes as well.

On August 10 the DC aircraft flew from Palmdale to Minneapolis. Figure 10 (left) is showing the plane altitude for the whole flight and on the right - the altitude and ratio plotted together while flying over Colorado. Because of the temperature changes on the previous flights for this one we were keeping the KTP crystals and the prefilter at a constant temperature. The prefilter temperature was $32.1 \mathrm{deg}$ C, the KTP temperature $33.9 \mathrm{deg} \mathrm{C}$ and FP temperature was $50.6 \mathrm{deg} \mathrm{C}$.
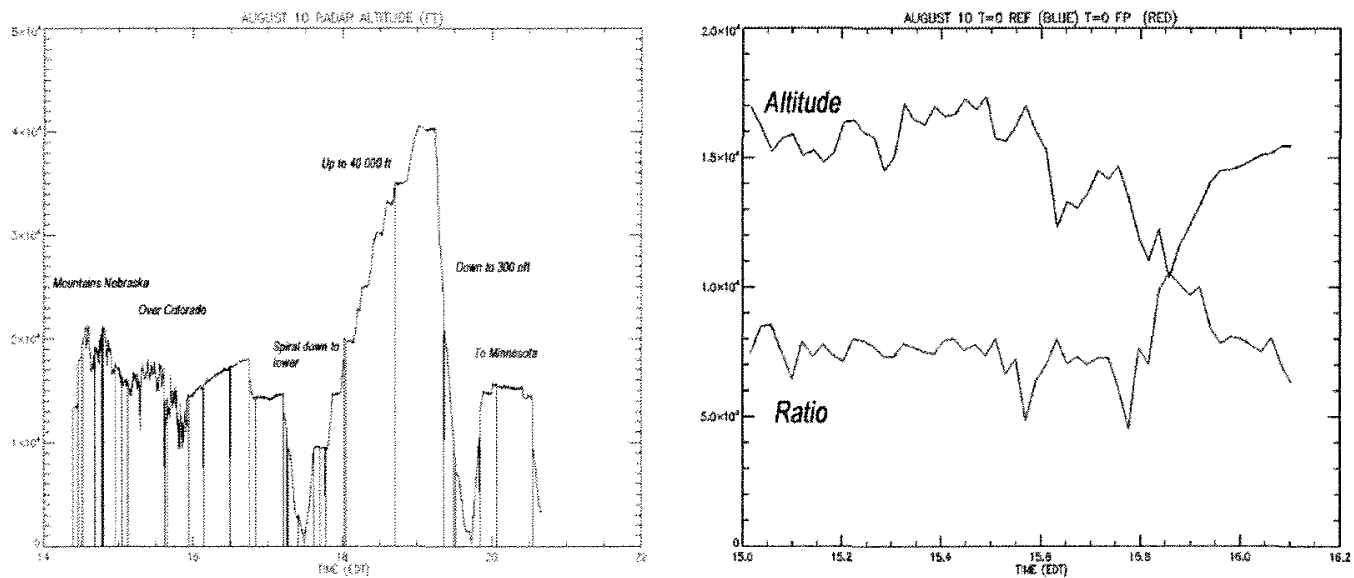

Figure 10.The altitude of the DC-8 aircraft (left) and the altitude and ratio plotted together (right) for the Colorado portion of the flight. We can see that when the plane is going up, the ratio is decreasing reflecting that the lidar is sensing a longer atmospheric column; more $\mathrm{CO}_{2}$ means smaller $\mathrm{FP}$ signal because of the absorption and as a result the ratio FP/Ref is decreasing. 

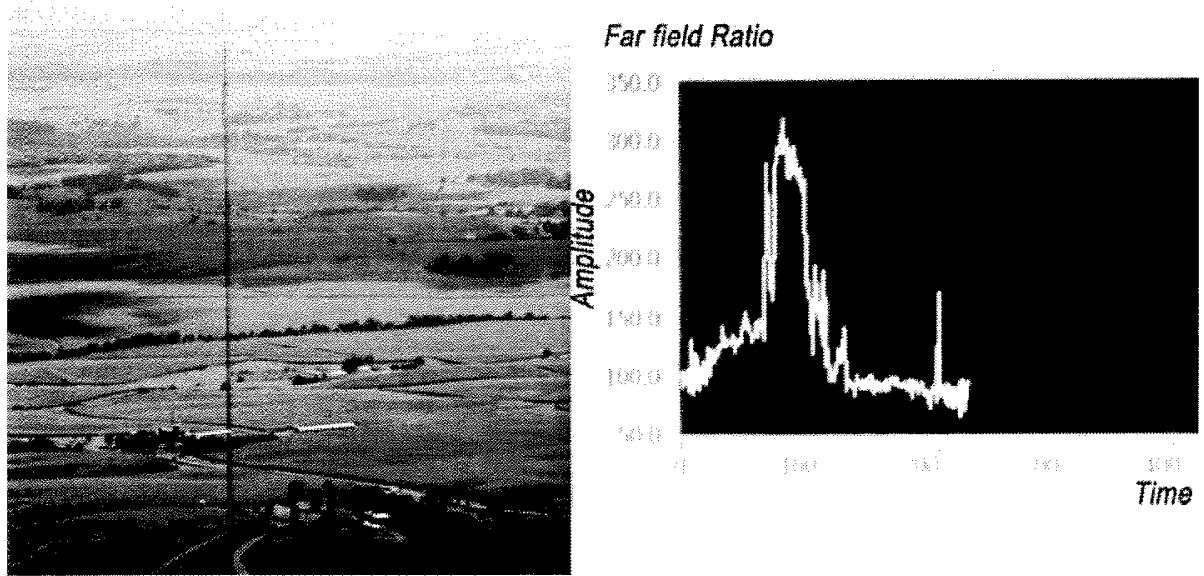

Figure 11. The amplitude far field ratio (right) is a measurement of $\mathrm{CO}_{2}$, the bell shape when going down and up around the WBTT tower (left) in Iowa shows that we can see $\mathrm{CO}_{2}$ change with our lidar. Spike after bell is because the plane is rapidly turning.

On August 11 we flew back from Minneapolis to Palmdale. There were a lot of clouds on this flight so the analysis algorithm has to differentiate between a ground return and a cloud return. Fig. 12 shows the lidar range obtained using our custom algorithm for finding the lidar peaks and the radar altimeter range. The good agreement illustrates that the algorithm is finding the correct peak. Range is plotted in feet.

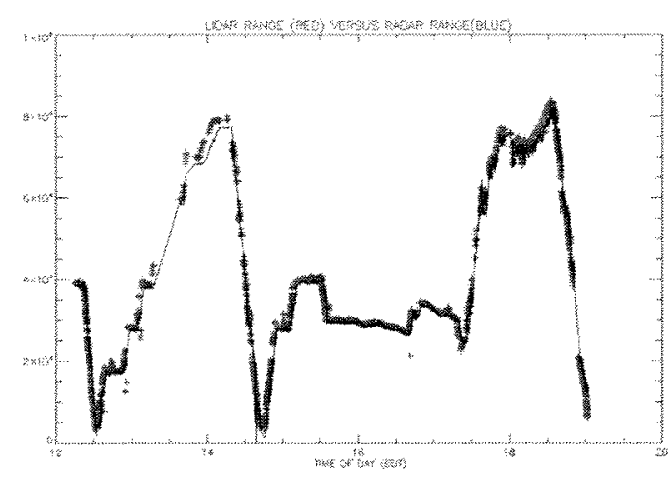

Figure 12. Lidar range (red) plotted with radar altimeter range (blue).

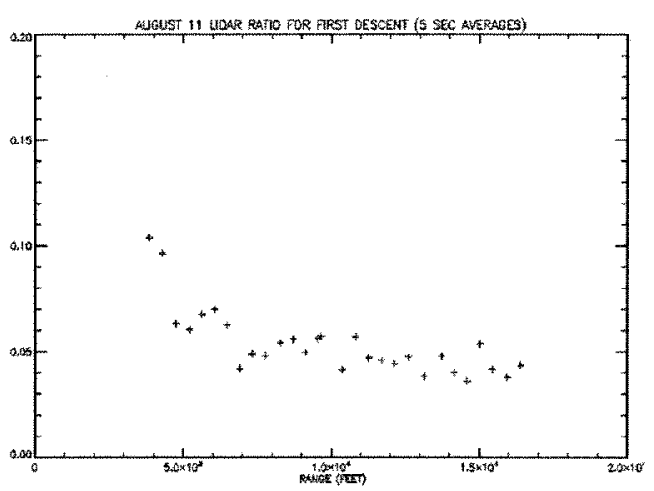

Figure 13. The FP/Ref ratio is changing during descent of the plane.

For the three descents on August 3, August 9 and August 11 the ratio changes vs altitude show repeatability.

From the plots we can calculate that for $6.1 \mathrm{~km} \Delta \mathrm{R}=0.105$, for $4.3 \mathrm{~km} \Delta \mathrm{R}=0.07$ and for $3.35 \mathrm{~km} \Delta \mathrm{R}=0.06$, and those numbers are consistent; however the instrument is not stable enough to measure with a precision of 
$1 \mathrm{ppm}$ yet. The data collected with the broadband lidar over different terrains will be further analyzed more precisely using retrieval algorithms and the results will be compared to the results of other researchers. The airborne campaign and the very preliminary analysis here provide an insight of how useful is the broadband technique and how the instrument can be further improved to become a candidate for NASA's ASCENDS mission.

\section{CONCLUSIONS}

The broad band lidar has succeeded in detecting $\mathrm{CO}_{2}$ from the DC- 8 aircraft. The major objective and our preliminary results show that the sensor is responding accordingly to changes of the aircraft altitude, demonstrating sensitivity, which could be scaled to a measurement from space. It exhibited strong signals at an altitude of $39,000 \mathrm{ft}$ as well as while flying above snow where the reflectance at 1.57 microns is less than $5 \%$. The system is still sensitive to misalignments that can be caused by aircraft vibration and temperature changes. Many factors including primarily cost induced us to make the choices for implementation that we made for the Broad Band Lidar. There are several aspects of the design which need to be optimized like the replacement of the off-the shelf bandpass prefilter with a custom-made one; the ovens, so we can better control the temperature of the optics and the receiving telescope. In this way we will improve signal-to-noise ratio (SNR) and the overall instrument performance.

The preliminary results over different terrain and under variety of atmospheric conditions give encouragement that we will be able to reach the required accuracy after making some necessary improvements.

\section{ACKNOWLEDGMENTS:}

This research was sponsored by NASA's Earth Science Technology Office. The authors want to thank John Burris for his help and support during the last part of the flight campaign.

\section{REFERENCES}

Abshire, J.B.et al, "Laser Sounder for Global Measurements of CO2 Concentrations from Space", 4th International Working Group on Greenhouse Gas Measurements from Space, (2007).

Abshire J. B., H. Riris, et al."Pulsed airborne lidar measurements of atmospheric CO2 column absorption," Tellus B $62770-783,(2010)$. 
Born, M., and E. Wolf, Principles of Optics, 360-371 pp., Cambridge University Press, Cambridge, 1999.

Browell E. V., J. Dobler, S. Kooi, Y. Choi, F. Harrison, B. Moore, and T. Zaccheo, "Airborne validation of active CO2 LAS measurements," EOS 90, A34C-04 (2009), Fall Meeting Supplement,abstract.

Browell, E.V., M.E. Dobbs, and B. Moore, Airborne Demonstration of a CW Laser System for CO2 Column Measurements, International Workshop on Greenhouse Gas Measurements from Space, (2007).

Chevallier F., Maksyutov S., Bousquet P.,Francois-Marie Breon, Saito R., Yoshida Y., and Yokota T, " On the accuracy of the $\mathrm{CO} 2$ surface fluxes to be estimated from the GOSAT observations", Geophysical Research Letters, vol. 36, (2009).

Chevallier F.,Francois-Marie Breon, and Rayner P., " Contribution of the Orbiting Carbon Observatory to the estimation of $\mathrm{CO} 2$ sources and sinks: Theoretical study in a variational data assimilation framework", Journal Of Geophysical Research, vol. 112, (2007).

Flamant Pierre H., "Atmospheric $\mathrm{CO}_{2}$ measurements by Lidar: an itinerary from ground to space," 4th International Working Group on Greenhouse Gas Measurements from Space, CNES HQ, Paris, June $27,(2007)$.

Georgieva E.M.,Wilson E.L., Miodek M., Heaps W.S., "Total Column Oxygen Detection Using Fabry-Perot Interferometer", Optical Engineering, 45(11), 115001, November, (2006).

Georgieva E.M., W. S. Heaps, E. L. Wilson.: "Differential Radiometers using Fabry-Perot Interferometric Technique for Remote Sensing of Greenhouse Gases", IEEE Transactions on Geoscience and Remote Sensing (TGARS), 46,10, October special issue, (2008).

Heaps, W.S., and S.R. Kawa, Fabry-Perot Interferometer for Column CO2, in NASA Earth Science Technology Conference, (2002).

Heaps, W.S., E.L. Wilson, Georgieva E.M, Precision Measurement of Atmospheric Trace Constituents Using a Compact Fabry-Perot Radiometer", International Journal of High Speed Electronics and Systems (IJHSES), 18, 3, September, (2008).

Heaps W.S., "Broadband lidar technique for precision CO2 measurement", Proc. SPIE, Vol. 7111, 711102, (2008).

Ingle, J.D., and S.R. Crouch, Spectrochemical Analysis, Prentice-Hall, Inc., New Jersey, (1988).

King, A.W., L. Dilling, G.P. Zimmerman et al., What Is the Carbon Cycle and Why Care? In: The First State of the Carbon Cycle Report (SOCCR): The North American Carbon Budget and Implications for the Global Carbon Cycle, A Report by the U.S. Climate Change Science Program and the Subcommittee on Global Change Research: National Oceanic and Atmospheric Administration, National Climatic Data Center, Asheville, NC, 15-20, (2007).

Koch G., J. Y. Beyon, F. Gibert, B. W. Barnes, S. Ismail, M. Petros, P. J. Petzar, J. Yu, E. A. Modlin, K. J, Davis, and U. N.Singh, "Side-line tunable laser transmitter for differential absorption lidar measurements of CO2: design and application to atmospheric measurements," Appl. Opt. 47, 944-956 (2008).

Neff BR, Dobbs ME, Gypson M, Pruitt J, Sharp WE, "CO2 monitoring with a field deployable NIR standoff environmental sensor", Proc. SPIE, Vol. 5154, 118-125, (2003).

NRC, Earth Science and Applications from Space: National Imperatives for the Next Decade and Beyond, The National Academies Press. Committee on Earth Science and Applications from Space: A Community Assessment and Strategy for the Future Space Studies Board, wwwnap edu, (2007).

Parkes AM, Keen KA, MeNaghten ED, "Trace gas detection using a novel cantilever-based photoacoustic spectrometer with multiplexed optical fiber-coupled diode lasers and fiber-amplification ",Proc. SPIE, Vol.6770, C7701-C7701, (2007).

Pruitt J, Dobbs ME, Gypson M, Neff BR, Sharp WE, "High-speed CW lidar retrieval using spectral lock-in algorithm", Proc. SPIE, Vol. 5154,138-145, (2003).

Rayner P.and O'Brien D., "The utility of remotely sensed $\mathrm{CO} 2$ concentration data in surface source inversions," Geophys.Res. Lett. 28,175-178 (2001).

Spiers G., Menzies R., Jacob et al., " Atmospheric $\mathrm{CO}_{2}$ measurements with a $2 \mu \mathrm{m}$ airborne laser absorption spectrometer employing coherent detection", Applied Optics, Vol. 50, No. 14, (2011).

Stultz R.D. and Ehritz M.E., "Temperature $(-32 \mathrm{degC}$ to $+90 \mathrm{degC})$ Performance of a $20 \mathrm{~Hz}$ Potassium Titanyl Phosphate Optical Parametric Oscillator", OSA TOPS on Advanced Solid-State Lasers, Vol.1, Stephen A.Payne and Clifford Pollock (eds.), (1996).

Wilson E.L., Georgieva E.M., Heaps W.S., "Development of a Fabry-Perot interferometer for ultra-precise measurements of column $\mathrm{CO}_{2}$ ", Meas. Sci. Technology, 18, 1495-1502, (2007). 